\title{
Physikalische Therapie: Extreme Kälte für schwere chronische Krankheiten
}

Jean-Michel Jeannin

Basel, Schweiz

\section{Einleitung}

Die physikalische Therapie definiert Kälte als Temperatur unter dem Thermoindifferenzpunkt. Der Thermoindifferenzpunkt ist die Temperatur eines Bades, bei dem keine Gegenregulation des Organismus ausgelöst wird. Eine besondere Form der Kältetherapie stellt die Kältekammer dar, in der der Patient für 1-3 min Temperaturen von $-70{ }^{\circ} \mathrm{C}$ oder $-120{ }^{\circ} \mathrm{C}$ ausgesetzt wird. Die analgetische Wirkung einer solchen Behandlung ist mit Experimenten nachgewiesen [1]. Nachfolgend wird eine Reihe von Publikationen besprochen, die sich mit der Kältetherapie von schweren, zum Teil invalidisierenden chronischen Krankheiten befassen.

\section{Multiple Sklerose}

Bei der Multiplen Sklerose (MS) handelt es sich um eine chronische, entzündliche Erkrankung des zentralen Nervensystems (ZNS). Charakteristisch sind zunehmende Lähmungen und auch Sehstörungen. Die Krankheit ist nicht heilbar, aber der Verlauf kann therapeutisch günstig beeinflusst werden. Pathogenetisch spielen durch das Immunsystem vermittelte Entzündungen sowie neurodegenerative Vorgänge wesentliche Rollen. Eine Reihe von Daten weist auf eine Beteiligung von oxidativem Stress an den degenerativen Prozessen hin [2]. Miller et al. [3] untersuchten in einer klinischen Studie den Einfluss einer Kältekammertherapie auf den antioxidativen Status des Plasmas sowie die Aktivität der beiden antioxidativen Enzyme Superoxid-Dismutase (SOD) und Katalase bei MSPatienten im Vergleich zu Gesunden. Patienten, die mit Immunmodulatoren, Immunstimulanzien, Vitaminen, Mineralstoffen oder sonstigen Substanzen mit antioxidativen Eigenschaften behandelt wurden, waren ausgeschlossen. Die Patienten der Testgruppe wurden im Ab- stand von 3 Monaten in drei Zyklen an $2 \times 5$ Tagen einer Woche (Montag-Freitag) in der Kältekammer behandelt. Während des dritten Zyklus erhielten die Patienten zusätzlich $10 \mathrm{mg}$ Melatonin täglich. Die Patienten der Kontrollgruppe wurden einem einzigen Behandlungszyklus in der Kältekammer unterzogen und erhielten kein Melatonin. Alle Parameter waren in der Kontrollgruppe nach der Kältekammerbehandlung unverändert. In der behandelten Gruppe zeigte sich eine Erhöhung des Gesamt-Antioxidantien-Status. Diese Erhöhung war nach der Gabe von Melatonin deutlich stärker ausgeprägt. Die Aktivität der SOD und der Katalase war nach den zwei ersten Zyklen unverändert, nach dem dritten Zyklus und der Gabe von Melatonin jedoch statistisch signifikant höher. Die Autoren folgern aus den Resultaten, dass eine Kältekammertherapie den oxidativen Stress bei MS-Patienten zu vermindern vermag, was den Verlauf der Krankheit günstig beeinflusst.

Dieselben Autoren untersuchten die Wirkung einer Kältekammertherapie auf den antioxidativen Status von 12 depressiven und 10 nicht depressiven MS-Patienten [4]. Es zeigte sich, dass nach der Kältekammerbehandlung der Antioxidantien-Spiegel signifikant angestiegen war. Bei den depressiven Patienten war der Spiegel stärker angestiegen als bei den nicht depressiven Patienten. Die Wirkungsweise einer Kältetherapie wird nach Ansicht der Autoren noch wenig verstanden und soll weiterhin erforscht werden.

\section{Kältebehandlung von schweren chronischen Schmerzzuständen}

Die Trigeminusneuralgie ist eine schwere, sehr schmerzhafte Störung im Gesicht. Als Hauptursache wird eine Kompression des Nervus trigeminus durch ein Blutgefäss angesehen [5]. Die Schmerzen können mit Anti-

\section{KARGER}

Fax +497614520714

\section{(c) 2017 S. Karger GmbH, Freiburg}

www.karger.com/szg 


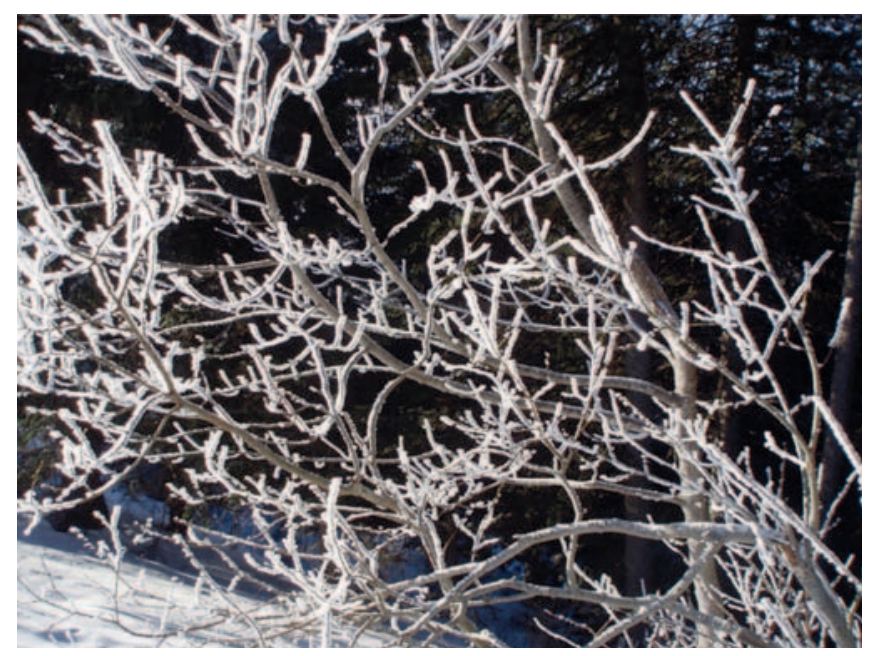

Abb. 1. Rigi, SZ, Januar 2011.

epileptika kontrolliert werden. In bestimmten Situationen, z.B. wenn die medikamentöse Therapie zu wenig wirksam oder mit schwerwiegenden Nebenwirkungen belastet ist, kann eine mikrochirurgische Dekompression des betroffenen Nervs durchgeführt werden [5].

29 Patienten mit einer paroxysmalen Trigeminusneuralgie wurden in insgesamt 83 Sitzungen kryotherapeutisch behandelt. Die Behandlung bestand in der intraoralen Anwendung von Kälte von $-120^{\circ} \mathrm{C}$ an den betroffenen freipräparierten Nerven am sedierten Patienten bei Lokalanästhesie. 85\% der Patienten waren nach der Behandlung schmerzfrei. Die langfristige Wirkung war weniger gut: $56,6 \%$ der Patienten waren weniger als 1 Jahr lang schmerzfrei, 25,3\% 1-2 Jahre und 4,8\% 5-6 Jahre. Die Patienten, die gut auf eine Behandlung ansprachen, zeigten in allen darauf folgenden Behandlungen ebenfalls gute Ergebnisse [6]. Die Methode ist mit einem Verlust an Sensibilität belastet, der aber in der Regel weniger lang dauert als die Schmerzfreiheit [7].

Die Fibromyalgie ist eine chronische invalidisierende schmerzhafte Entzündung des Bewegungsapparats, namentlich der Muskeln. Die Patienten sind im Alltag stark eingeschränkt, die Lebensqualität ist stark beeinträchtigt. Die starken Schmerzen entstehen im Zusammenhang mit einer Störung der Verarbeitung von sensiblen Afferenzen im ZNS. Die Störung hat zur Folge, dass Stimuli, die normalerweise nicht als schmerzhaft empfunden werden, als Schmerzen wahrgenommen werden (Allodynie). Auf molekularer Ebene werden ein Ungleichgewicht unter den Neurotransmittern und eine Zunahme von an Entzündungsvorgängen beteiligten Substanzen diskutiert [8].

Die Wirksamkeit einer Kältekammerbehandlung wurde in einer Studie mit 100 ambulanten Fibromyalgie-Patienten untersucht. 50 Patienten erhielten eine Kältekammertherapie, 50 Patienten bildeten die Kontrollgruppe. Die Gruppen waren bezüglich der Altersverteilung sehr homogen. Alle Teilnehmer behielten die vom Hausarzt

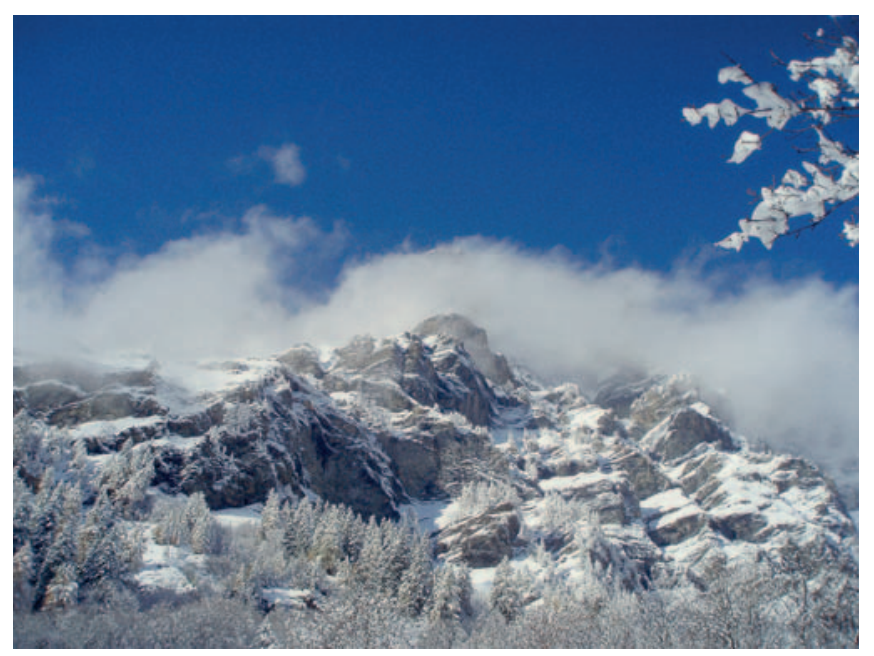

Abb. 2. Leukerbad, VS, Oktober 2008.

verschriebene medikamentöse Behandlung bei. Die Kältekammerbehandlung bestand aus total 15 Sitzungen, verteilt auf die ersten 5 Tage einer Woche während 3 Wochen. Nach einer Anpassung von $30 \mathrm{~s}$ bei $-60^{\circ} \mathrm{C}$ verweilten die Patienten 3 min bei $-140^{\circ} \mathrm{C}$. Anschliessend folgten 30 min Bewegung auf dem Fahrradergometer oder in der Tretmühle. Die in der Kältekammer behandelten Patienten berichteten über eine gegenüber der Kontrollgruppe deutlich stärkere Verbesserung der Lebensqualität. Die Autoren vermuten, dass die Kälte direkt auf das Gleichgewicht zwischen entzündungsfördernden und entzündungshemmenden Mediatoren einwirkt [8].

Von einer Abnahme von Entzündungsmarkern bei Patienten mit ankylosierender Spondylitis (Morbus Bechterew) und bei Gesunden nach Behandlung in der Kältekammer berichten Stanek et al. [9]. Der Morbus Bechterew ist eine chronisch-entzündliche rheumatische Krankheit, die zur Invalidisierung führen kann.

\section{Kältekammer bei Depression und Angstzuständen}

Die Wirksamkeit einer Behandlung in der Kältekammer bei Patienten mit nachgewiesener Depression oder nachgewiesenem Angstzustand wurde anhand der Veränderung des Schweregrads der Krankheit gemäss der Hamilton-Depressionsskala (16 Kriterien) bzw. der Hamilton-Angstskala (14 Kriterien) sowie anhand einer Selbstbeurteilungsskala für Lebensqualität bewertet. Insgesamt wurden 26 Patienten behandelt; 34 Patienten bildeten die Kontrollgruppe. Die Behandlung in der Kältekammer wurde zusätzlich zur medikamentösen Therapie verabreicht, welche unverändert weitergeführt wurde. Die medikamentöse Basis-Therapie bestand aus Thymoleptika, Benzodiazepinen und Neuroleptika - einzeln oder kombiniert. Sie wurde im Rahmen der Studie unverändert 
weitergeführt. Die geprüfte Behandlung bestand in 15 Kryotherapie-Sitzungen von $2-3$ min Dauer bei $-110^{\circ} \mathrm{C}$ bis $-160^{\circ} \mathrm{C}$, verteilt auf 3 Wochen. 12 von 16 Kriterien der Hamilton-Skala, hiervon 14 signifikant, und 11 von 14 Kriterien der Angstskala, davon 8 signifikant, zeigten eine Verbesserung. Die Lebensqualität gemäss Selbstbeurteilung der Patienten war nach der Behandlung in der Kältekammer für alle 11 Kriterien verbessert, wovon das Ergebnis für 6 Kriterien statistisch signifikant war. Die Autoren gelangten zu dem Schluss, dass eine Kältekammertherapie zur Unterstützung einer medikamentösen antidepressiven oder anxiolytischen Therapie nützlich sei. Über die Wirkungsweise äusserten sich die Autoren nicht. Kritisch muss Folgendes erwähnt werden: Die beiden Gruppen der Studienpopulation unterschieden sich betreffend einiger Kriterien in signifikantem Mass, z.B. Schlafstörungen, Verhalten bei der Befragung, Störungen der Sexualfunktion. Die medikamentöse Basis-Therapie war uneinheitlich, wurden doch sehr verschiedene Medikamente (Antidepressiva und Neuroleptika) verabreicht [10]. Somit wurde eine Reihe von Faktoren, die einen Einfluss auf das Ergebnis haben könnten, nicht berücksichtigt. Da für die Mehrheit der untersuchten Kriterien ein statistisch signifikantes Resultat beobachtet wurde, kann der Folgerung der Autoren dennoch zugestimmt werden.

Mit einer weniger drastischen Behandlung in Form von kalten Duschen $\left(20^{\circ} \mathrm{C}\right.$ während 2-3 min), die nach einem bestimmten Plan appliziert wurden, konnte eine antidepressive Wirkung bei depressiven Patienten erzielt werden [11].

\section{Reflexionen}

Das Therapiemittel Kälte eignet sich sehr gut zur Behandlung von Schmerzen bei entzündlichen und degenerativen Krankheiten sowie Verletzungen des Bewegungsapparats. Lokale Kälte drosselt die Nervenleitgeschwindigkeit, senkt die Schmerzempfindlichkeit, hemmt die Entzündung und schwellt das Gewebe ab. Für generalisierte Schmerzen und für multilokale rheumatische Erkrankungen eignet sich die Ganzkörpertherapie in der Kältekammer [1]. Bisweilen wird Kälte auch mikrochirurgisch angewendet - grundsätzlich mit der gleichen Wirkung von Entzündungshemmung und Analgesie. Adäquat angewendet ist das Therapiemittel Kälte eine wertvolle Ergänzung in der Behandlung von chronisch-entzündlichen schmerzhaften Krankheiten, bisweilen auch eine vernünftige Alternative. Die in dieser Arbeit ebenfalls beschriebene Behandlung von Depressionskranken in der Kältekammer scheint wenig Resonanz zu finden. Es wurde auch kein molekularer Mechanismus für die Wirksamkeit der Methode gefunden. Eine Depression trifft das Zentrum eines Menschen, wo es keine messbaren Parameter gibt. Zur Prüfung der Wirksamkeit therapeutischer Interventionen bei Depressionen bedarf es adäquater Instrumente, die auch den Einfluss der Intervention selber erfassen und - warum auch nicht? - Placeboeffekte berücksichtigen.

\section{Literatur}

1 Gutenbrunner C: 3.8 Wärme- und Kälteträgertherapie; in Gutenbrunner C, Glaesener JJ: Rehabilitation, Physikalische Medizin und Heilverfahren. Heidelberg, Springer, 2007, p 75.

2 Gilgun-Sherki $Y$, et al: The role of oxidative stress in the pathogenesis of multiple sclerosis. J Neurol 2004;251:261-268.

3 Miller E, et al: The effects of whole-body cryotherapy on oxidative stress in multiple sclerosis patients. J Therm Biol 2010;35:406410.

4 Miller E, et al: Effects of whole-body cryotherapy on a total antioxidative status and activities of antioxidative enzymes in blood of depressive multiple sclerosis patients. World J Biol Psychiatry 2011;12:223-227.
5 Steiger HJ, et al: Therapiemöglichkeiten der Trigeminusneuralgie - Chirurgische Verfahren im Fokus. Dtsch Arztebl 2007;104:A2655A2661 + A1-A2.

6 Zakrzewska JM: Cryotherapy in the management of paroxysmal trigeminal neuralgia. J Neurol Neurosurg Psychiatry 1987;50:485487.

7 Barnard D, et al: Cryoanalgesia in the management of chronic facial pain. J Maxillofac Surg 1981;9:101-102.

8 Bettoni L, et al: Effects of 15 consecutive cryotherapy sessions on the clinical output of fibromyalgic patients. Clin Rheumatol 2013;32: 1337-1345.
9 Stanek A, et al: influence of cryogenic temperatures on inflammatory markers in patients with ankylosing spondylitis. Polish J Environ Stud 2010;19:167-175.

10 Rymaszewska J, Ramsey D: Whole blood cryotherapy as a novel adjuvant therapy for depression and anxiety. Arch Psychiatry Psychotherapy 2008;2:49-57.

11 Shevchuk N: Adapted cold shower as potential treatment for depression. Med Hypotheses 2008;70:995-1001. 